\title{
On the Erdős-Turán inequality for balls
}

\author{
by
}

\author{
Glyn Harman (Cardiff)
}

1. Introduction. Let $(\Omega, \mathcal{M}, \mu)$ be a probability space. Given a family of sets $\mathcal{F} \subseteq \mathcal{M}$, we define the discrepancy of a set of points $\left\{x_{1}, \ldots, x_{N}\right\} \subseteq X$ with respect to $\mathcal{F}$ by

$$
D_{N}\left(\mathcal{F}, x_{n}\right)=\sup _{\mathcal{A} \in \mathcal{F}}\left|\sum_{\substack{x_{n} \in \mathcal{A} \\ n \leq N}} 1-N \mu(\mathcal{A})\right| .
$$

The most well known case of this has $\Omega=\mathbb{T}=\mathbb{R} / \mathbb{Z}, \mu(\cdot)$ the usual Haar measure (equivalent to Lebesgue measure on $[0,1)$ ) and $\mathcal{F}$ the family of intervals $(\bmod 1)$. For this case Erdős and Turán [4] proved the following upper bound:

$$
D_{N}\left(\mathcal{F}, x_{n}\right) \leq \frac{C_{1} N}{L}+C_{2} \sum_{m=1}^{L} \frac{1}{m}\left|\sum_{n=1}^{N} e\left(m x_{n}\right)\right|
$$

for all positive integers $L$, where $e(y)=\exp (2 \pi i y)$. Here $C_{1}$ and $C_{2}$ are absolute, and the best known values are $C_{1}=1, C_{2}=2+2 / \pi$ (see [1], p. 20, or [10], p. 8). The Erdős-Turán theorem was generalized independently to $\mathbb{T}^{k}$ by Koksma [9] and Szüsz [13]. They took $\mathcal{F}$ to be the family of boxes with sides parallel to the axes (see also [3] and [6], §5.4). It is possible to prove an analogue when $\mathcal{F}$ is the family of all boxes. As is well known, allowing the boxes to be "tilted" greatly increases the discrepancy in general (see [6], $\S 5.4,[2]$ and [10]).

In [7] Holt has given an Erdős-Turán inequality for balls. This was based on an approximation to the characteristic function of a ball which is in some sense optimal [8]. It is the purpose of this note to prove a stronger version of this result. It may seem paradoxical that we will not employ the "optimal" functions used by Holt. We will explain below the difference between Holt's work and our own. Suffice it to say now that his approach optimized the analogue of $C_{1}$ above, but gave the wrong size coefficients in the analogue

1991 Mathematics Subject Classification: Primary 11K38. 
of the sum over $m$. We will not try to optimize $C_{1}$ and $C_{2}$, but we will get the correct order of diminution for the coefficients. If we write $\mathcal{B}(r)$ for the family of balls with radius $r$, Holt's result is as follows.

Theorem 1. Let $r \geq 0$ and $\left\{\mathbf{x}_{1}, \ldots, \mathbf{x}_{N}\right\} \subset \mathbb{T}^{k}$. Then, for all $s>0$,

$$
\begin{aligned}
& D_{N}\left(\mathcal{B}(r), \mathbf{x}_{n}\right) \leq N A_{k}(r, s) \\
& \quad+\sum_{0<|\mathbf{m}|<s}\left(A_{k}(r, s)+\left(\frac{r}{|\mathbf{m}|}\right)^{k / 2}\left|J_{k / 2}(2 \pi|\mathbf{m}|)\right|\right)\left|\sum_{n=1}^{N} e\left(\mathbf{m} \cdot \mathbf{x}_{n}\right)\right| .
\end{aligned}
$$

In the above $J_{\nu}$ denotes the Bessel function of order $\nu$, and, when $r s>1$,

$$
A_{k}(r, s) \ll r^{k-1} s^{-1} \text {. }
$$

The fault with (1.2) is that the coefficients involving $\mathbf{m}$ should have size

$$
\left(\frac{r}{|\mathbf{m}|}\right)^{k / 2}\left|J_{k / 2}(2 \pi|\mathbf{m}|)\right| \ll \min \left(r^{k}, \frac{r^{(k-1) / 2}}{|\mathbf{m}|^{(k+1) / 2}}\right) .
$$

Our main result establishes this. We will write $C_{j}(t), C_{j}(a, b)$, etc. for constants depending only on given parameters $(t, a, b$ in the examples quoted).

TheOREM 2. Under the hypotheses of Theorem 1 we have

$$
\begin{aligned}
& D_{N}\left(\mathcal{B}(r), \mathbf{x}_{n}\right) \leq C_{1}(k) N\left(r^{k-1} s^{-1}+s^{-k}\right) \\
& \quad+C_{2}(k) \sum_{0<|\mathbf{m}|<s}\left(\frac{1}{s^{k}}+\min \left(r^{k}, \frac{r^{(k-1) / 2}}{|\mathbf{m}|^{(k+1) / 2}}\right)\right)\left|\sum_{n=1}^{N} e\left(\mathbf{m} \cdot \mathbf{x}_{n}\right)\right| .
\end{aligned}
$$

In particular, writing $\mathcal{C}$ for the family of all balls of radius less than one half, we have

$$
D_{N}\left(\mathcal{C}, \mathbf{x}_{n}\right) \leq C_{3}(k) \frac{N}{s}+C_{4}(k) \sum_{0<|\mathbf{m}|<s} \frac{1}{|\mathbf{m}|^{(k+1) / 2}}\left|\sum_{n=1}^{N} e\left(\mathbf{m} \cdot \mathbf{x}_{n}\right)\right| .
$$

We state two applications of the above result as further theorems.

TheOREM 3. Let $p$ be a prime number. Then there is a lattice point $\mathbf{h} \in \mathbb{Z}^{k}$ such that $|\mathbf{h}|<p$ and the sequence $\mathbf{x}_{n}=n \mathbf{h} / p$ satisfies, for all $r \in(0,1)$,

$$
D_{p}\left(\mathcal{B}(r), \mathbf{x}_{n}\right) \ll\left(p r^{k}\right)^{(k-1) /(k+1)}+1 .
$$

TheOrem 4. For almost all $\boldsymbol{\alpha} \in \mathbb{T}^{k}$ we have

$$
D_{N}(\mathcal{C}, n \boldsymbol{\alpha}) \ll N^{1-2 /(k+1)} \log ^{2} N .
$$

We note that in $(1.5)$ we have improved Holt's exponent $(k-1) / k$ (Theorem 2 of [7]). We have also relaxed the condition $r^{k} p>1$, but this only involved the addition of the term 1 in (1.5). We remark that the corresponding bounds for tilted boxes have the form $N^{1-1 / k}$, a result which is known 
to be best possible (see [14] and $\S 5.4$ of [6]). The author has not been able to determine whether (1.5) and (1.6) are quite sharp in the case $k=2$ (exponent $1 / 3$ ), or whether the universal lower bound for (1.6)

$$
D_{N}\left(\mathcal{C}, \mathbf{x}_{n}\right) \gg N^{1 / 4}
$$

may be nearer the truth.

2. Preparatory lemmas. A real even entire function is an entire function which is even and only takes real values on the real axis. Let $\mathcal{E}(s)$ be the set of all such functions which satisfy

$$
f(z)=O(\exp (2 \pi s|z|)) \quad \text { as }|z| \rightarrow \infty .
$$

Lemma 1. Let $r, s>0$. Then there are functions $F_{1}(z), F_{2}(z) \in \mathcal{E}(s)$ such that

$$
F_{1}(x) \leq \chi(x) \leq F_{2}(x), \quad x \in \mathbb{R},
$$

where $\chi(x)$ is the characteristic function of the interval $[-r, r]$, and

$$
F_{j}(x)=\chi(x)+O\left(\min \left(1, \frac{1}{|s(x-r)|^{2}}\right)\right),
$$

where the constant implied by the $O$ notation is absolute.

P r o of. See [8], Chapter 1 of [10], or Chapter 2 of [1]. Usually the closeness of approximation of $F_{j}(x)$ to $\chi(x)$ is given by calculating

$$
\int_{-\infty}^{\infty}\left(F_{2}(x)-F_{1}(x)\right) d x \quad \text { or } \quad \int_{-\infty}^{\infty}\left|F_{j}(x)-\chi(x)\right| d x .
$$

The estimate (2.2) will prove more helpful in our context for reasons which should become clear.

Lemma 2. Let $r, s>0$ and $k \in \mathbb{N}$. Then there are functions $G_{1}(z), G_{2}(z)$ $\in \mathcal{E}(s)$ such that

$$
G_{1}(x) \leq \chi(x) \leq G_{2}(x)
$$

and

$$
\left|G_{j}(x)-\chi(x)\right| \leq C(k) h(x),
$$

where

$$
h(x)=\min \left(1,|s(x-r)|^{-2 j(x, r)}\right), \quad j(x, r)= \begin{cases}k & \text { if }|x| \geq r, \\ 1 & \text { if }|x|<r .\end{cases}
$$

Pr o of. We appeal to Lemma 1 with $s$ replaced by $s / k$. We take $G_{2}(x)=$ $F_{2}(x)^{k}$, which clearly satisfies all our demands. For odd $k$ we can take 
$G_{1}(x)=F_{1}(x)^{k}$. For $k$ even, either replace $k$ by $k+1$ (since the truth of (2.5) with $k+1$ implies its truth for $k$ ), or take

$$
G_{1}(x)=k F_{1}(x) F_{2}(x)^{k-1}-(k-1) F_{2}(x)^{k} .
$$

See Lemma 6 of [5] for the motivation and proof that this is a lower bound function.

REMARK. The advantage of the functions constructed above is that the errors are given a suitable bound both for small and large values of $x$, unlike the situation in [8] where the term of importance is

$$
\int_{0}^{\infty}\left|F_{j}(x)-\chi(x)\right| x^{u} d x,
$$

and the $x^{u}$ factor cancels out the errors for small $x$. To obtain our results, essentially we will need to estimate such integrals for two different values of $u$, as becomes clear in the next lemma.

Lemma 3. Let $r, s>0$ and $k \in \mathbb{N}$. Let $G_{1}(x), G_{2}(x)$ be the functions given by Lemma 2. Then, for all $m$ with $1 \leq m \leq 2 k-1$, and all $v$ with $0<v \leq s$, we have

$$
\begin{aligned}
& \int_{0}^{\infty}\left|G_{j}(x)-\chi(x)\right| \min \left(x^{m-1},\left(\frac{x}{v}\right)^{(m-1) / 2}\right) d x \\
& \leq C_{j}(k, m)\left(\frac{1}{s^{m}}+\min \left(r^{m}, \frac{r^{(m-1) / 2}}{v^{(m+1) / 2}}\right)\right)
\end{aligned}
$$

for $j=1,2$. Moreover,

$$
\int_{0}^{\infty}\left|G_{j}(x)-\chi(x)\right| x^{m-1} d x \leq C_{j+2}(k, m)\left(\frac{1}{s^{m}}+\frac{r^{m-1}}{s}\right) .
$$

Proof. These results follow from Lemma 2 after splitting $[0, \infty)$ into appropriate regions. For example, if $v>1 / r$ we have to consider, for (2.6), the four integrals (assuming $v^{-1}+s^{-1}<r$ for clarity)

$$
\begin{array}{ll}
\int_{0}^{1 / v} \frac{x^{m-1}}{s^{2}(r-x)^{2}} d x, & \int_{1 / v}^{r-1 / s}\left(\frac{x}{v}\right)^{(m-1) / 2} \frac{1}{s^{2}(r-x)^{2}} d x, \\
\int_{r-1 / s}^{r+1 / s}\left(\frac{x}{v}\right)^{(m-1) / 2} d x, & \int_{r+1 / s}^{\infty}\left(\frac{x}{v}\right)^{(m-1) / 2} \frac{1}{s^{2 k}(x-r)^{2 k}} d x .
\end{array}
$$

The reader will have no difficulty in verifying that each of these integrals has the right size. 
3. Proof of Theorem 2. Theorem 2 follows immediately from the following lemma which may have other applications. We write $V_{k}$ for the volume of a ball belonging to $\mathcal{B}(1)$ in $\mathbb{R}^{k}$.

Lemma 4. Let $\mathcal{B} \in \mathcal{B}(r)$ in $\mathbb{T}^{k}$, and let $\chi(\mathbf{x})$ be its characteristic function. Let $s>0$. Then there are functions $H_{1}(\mathbf{x}), H_{2}(\mathbf{x})$ such that

$$
H_{1}(\mathbf{x}) \leq \chi(\mathbf{x}) \leq H_{2}(\mathbf{x})
$$

and

$$
H_{j}(\mathbf{x})=V_{k} r^{k}+O\left(\frac{1}{s^{k}}+\frac{r^{k-1}}{s}\right)+\sum_{0<|\mathbf{m}|<s} c_{j}(\mathbf{m}) e(\mathbf{m} \cdot \mathbf{x})
$$

where

$$
\left|c_{j}(\mathbf{m})\right| \ll \frac{1}{s^{k}}+\min \left(r^{k}, \frac{r^{(k-1) / 2}}{|\mathbf{m}|^{(k+1) / 2}}\right) .
$$

Proof. We deal with the construction of $H_{1}(\mathbf{x})$ only; the choice of $H_{2}$ is analogous. Also, without loss of generality, we can suppose that $\mathcal{B}$ is centred at the origin: this only affects $c_{j}(\mathbf{m})$ by a factor $e(-\mathbf{m} \cdot \mathbf{a})$, where $\mathbf{a}$ is the centre of the ball. We pick $G_{1}(x)$ from Lemma 2 , and let $\chi(x)$ be as defined in Lemma 1. There should be no confusion in the following between $\chi(\mathbf{x})$ the characteristic function of the ball, and $\chi(x)$ the characteristic function of the interval. Since $G_{1}$ is an even entire function we may write

$$
G_{1}(x)=\sum_{n=0}^{\infty} c_{n} x^{2 n} .
$$

Also, if we write $\mathbf{z}=\left(z_{1}, \ldots, z_{k}\right)$, the function

$$
U(\mathbf{z})=\sum_{n=0}^{\infty} c_{n}\left(z_{1}^{2}+\ldots+z_{k}^{2}\right)^{n}
$$

is an entire function of $k$ complex variables, and

$$
|U(\mathbf{z})|=O(\exp (2 \pi s\|\mathbf{z}\|))
$$

where

$$
\|\mathbf{z}\|=\sup _{\mathbf{x} \in \mathcal{S}}|\mathbf{z} \cdot \mathbf{x}|,
$$

and $\mathcal{S} \in \mathcal{B}(1)$ is centred at the origin (see [8] for details).

Write

$$
\widehat{U}(\mathbf{y})=\int_{\mathbb{R}^{k}} U(\mathbf{x}) e(-\mathbf{x} \cdot \mathbf{y}) d \mathbf{x} .
$$

Then, using (3.4) with Theorem 4.9 of Chapter 3 in [12], we obtain $\widehat{U}(\mathbf{y})=0$ 
for $|\mathbf{y}| \geq s$. Also

$$
\begin{aligned}
\widehat{U}(\mathbf{0}) & =\int_{\mathbb{R}^{k}} U_{1}(\mathbf{x}) d \mathbf{x}=k V_{k} \int_{0}^{\infty} G_{1}(t) t^{k-1} d t \\
& =k V_{k} \int_{0}^{\infty} \chi(t) t^{k-1} d t-\int_{0}^{\infty}\left(\chi(t)-G_{1}(t)\right) t^{k-1} d t \\
& =V_{k} r^{k}+O\left(\frac{r^{k-1}}{s}+\frac{1}{s^{k}}\right)
\end{aligned}
$$

by Lemma 3. Moreover,

$$
\begin{aligned}
\widehat{U}(\mathbf{v}) & =\int_{\mathbb{R}^{k}} \chi(\mathbf{x}) e(-\mathbf{x} \cdot \mathbf{v}) d \mathbf{x}-\int_{\mathbb{R}^{k}}(\chi(\mathbf{x})-U(\mathbf{x})) e(-\mathbf{x} \cdot \mathbf{v}) d \mathbf{x} \\
& =I_{1}(\mathbf{v})-I_{2}(\mathbf{v}) \quad \text { say. }
\end{aligned}
$$

Now, as is well known (it follows from Theorem 3.3 of [12], Chapter 4, for example),

$$
I_{1}(\mathbf{v})=\left(\frac{r}{|\mathbf{v}|}\right)^{k / 2} J_{k / 2}(2 \pi|\mathbf{v}| r) .
$$

Also, since $\chi$ and $G_{1}$ are both radial functions, we can apply Theorem 3.3 of [12], Chapter 4 , to $I_{2}$ to obtain

$$
I_{2}(\mathbf{v})=2 \pi|\mathbf{v}|^{1-k / 2} \int_{0}^{\infty}\left(\chi(x)-G_{1}(x)\right) J_{k / 2-1}(2 \pi|\mathbf{v}| x) x^{k / 2} d x .
$$

Since, for $u>0$,

$$
J_{u}(x) \leq C(u) \min \left(x^{-1 / 2}, x^{u}\right) \quad \text { for } x>0,
$$

(3.6) and (3.7) with Lemma 3 (with $m=k$ say) give, for $|\mathbf{v}|>0$,

$$
|\widehat{U}(\mathbf{v})| \ll\left(\frac{1}{s^{k}}+\min \left(r^{k}, \frac{r^{(k-1) / 2}}{|\mathbf{v}|^{(k+1) / 2}}\right)\right) .
$$

Hence, if we write

$$
H_{1}(\mathbf{x})=\sum_{\mathbf{n} \in \mathbb{Z}} U(\mathbf{x}+\mathbf{n}),
$$

we have (3.1)-(3.3) as desired by the $k$-dimensional Poisson summation formula (see [12], pp. 251-252, for example).

4. Proof of Theorem 3. By the argument on page 65 of [7] there is a lattice point $\mathbf{h}$ with $|\mathbf{h}|<p$, and, using our Theorem 2 in place of Holt's 
Theorem 1, we obtain

$$
\begin{aligned}
D_{p}\left(\mathcal{B}(r), \mathbf{x}_{n}\right) & \ll p s^{-1} r^{k-1}+\sum_{|\mathbf{m}|<s} \min \left(r^{k}, \frac{r^{(k-1) / 2}}{|\mathbf{m}|^{(k+1) / 2}}\right) \\
& \ll p s^{-1} r^{k-1}+1+(r s)^{(k-1) / 2}
\end{aligned}
$$

after an easy calculation. If we then take the optimal choice

$$
s=\left(p^{2} r^{k-1}\right)^{1 /(k+1)}
$$

to balance the first and last terms in (4.1) we thereby obtain (1.5).

5. Proof of Theorem 4 . We note that, if $\|\cdot\|$ now denotes distance to a nearest integer, then

$$
\left|\sum_{n \leq N} e(n \boldsymbol{\alpha} \cdot \mathbf{m})\right| \leq \min \left(N, \frac{1}{\|\boldsymbol{\alpha} \cdot \mathbf{m}\|}\right) .
$$

If $\mathbf{m}=\left(m_{1}, \ldots, m_{k}\right)$, write

$$
d(\mathbf{m})=\prod_{j=1}^{k} \max \left(1,\left|m_{j}\right|\right) .
$$

By Theorem 2 of [11] (applied in $1, \ldots, k$ dimensions), we have, for almost all $\boldsymbol{\alpha}$ and all $\varepsilon>0$,

$$
\sum_{0<|\mathbf{m}|<s}(d(\mathbf{m})\|\boldsymbol{\alpha} \cdot \mathbf{m}\|)^{-1} \ll(\log s)^{k+1+\varepsilon} .
$$

From this it easily follows that, for almost all $\boldsymbol{\alpha}$,

$$
\sum_{0<|\mathbf{m}|<s}\left(|\mathbf{m}|^{(k+1) / 2}\|\boldsymbol{\alpha} \cdot \mathbf{m}\|\right)^{-1} \ll s^{(k-1) / 2}(\log s)^{k+1+\varepsilon} .
$$

Theorem 4 then follows from Theorem 2 with the choice

$$
s=N^{2 /(k+1)}(\log N)^{-\varepsilon-2} .
$$

\section{References}

[1] R. C. Baker, Diophantine Inequalities, Oxford Univ. Press, 1986.

[2] J. Beck and W. W. L. Chen, Irregularities of Distribution, Cambridge Univ. Press, 1987.

[3] T. Cochrane, Trigonometric approximation and uniform distribution modulo 1 , Proc. Amer. Math. Soc. 103 (1988), 695-702.

[4] P. Erdös and P. Turán, On a problem in the theory of uniform distribution, I, Indag. Math. 10 (1948), 370-378.

[5] G. Harman, Small fractional parts of additive forms, Philos. Trans. Roy. Soc. London Ser. A 345 (1993), 339-347. 
[6] G. Harman, Metric Number Theory, Oxford Univ. Press, 1998.

[7] J. J. Holt, On a form of the Erdős-Turán inequality, Acta Arith. 74 (1996), 61-66.

[8] J. J. Holt and J. D. Vaaler, The Beurling-Selberg extremal functions for a ball in Euclidean space, Duke Math. J. 83 (1996), 203-248.

[9] J. F. Koksma, Some theorems on Diophantine inequalities, Math. Centrum Amsterdam Scriptum no. 5.

[10] H. L. Montgomery, Ten Lectures on the Interface Between Analytic Number Theory and Harmonic Analysis, Amer. Math. Soc., Providence, R.I., 1994.

[11] W. M. Schmidt, Metrical theorems on fractional parts of sequences, Trans. Amer. Math. Soc. 110 (1964), 493-518.

[12] E. M. Stein and G. Weiss, Introduction to Fourier Analysis on Euclidean Spaces, Princeton Univ. Press, Princeton, N.J., 1971.

[13] P. Szüsz, Über ein Problem der Gleichverteilung, in: Comptes Rendus du Premier Congrès des Mathématiciens Hongrois, 1950, 461-472.

[14] S. K. Zaremba, Good lattice points in the sense of Hlawka and Monte Carlo integration, Monatsh. Math. 72 (1968), 264-269.

School of Mathematics

Cardiff University

P.O. Box 926

Cardiff CF2 4YH

Wales, U.K.

E-mail: Harman@cf.ac.uk 